\title{
Tricritical behavior of random systems with a coupling to a nonfluctuating parameter
}

\author{
Y.N.Skryabin ${ }^{1}$ \\ Institute for Metal Physics, Russian Academy of Sciences, \\ Ural Division, Kovalevskaya Str., 18, \\ 620219, Ekaterinburg, Russia \\ A.V.Shchanov \\ Ural State Technical University, \\ Mir Str., 19, 620002, Ekaterinburg, Russia
}

\begin{abstract}
The influence of disordering upon critical behavior of the system with hidden degrees of freedom is considered. It is shown that there is a tricritical behavior in the constrained system, while in the unconstrained system only phase transitions of the second order occur.
\end{abstract}

Key words: Magnetically ordered materials. Point defects. Phase transitions.

\section{Introduction}

Recently, phase transitions under a constraint imposed on some "hidden" degrees of freedom coupled to the critical ones have again attracted attention [1]. As it is well known, many real systems possess a hidden degree of freedom [2-4], which can be described as the nonfluctuating parameter in the vicinity of the phase transition. Fisher [5] stressed the importance of the constraint upon the hidden degree of freedom. Fisher's theory of phase transition in constrained systems was generalized $[6,7]$ to include the possibility of first- order

$\overline{1}$ Partially supported by State Program "Actual Problems in Condensed Matter Physics: Neutron Studies"(Projects No. 96-104, 96-305) and Russian Foundation for Basic Research (Project No. 97-02-17315), Russia. 
phase transitions. In particular, it was found that the tricritical behavior coincides with the critical behavior of the ideal system provided $\alpha>0$ ( $\alpha$ is the critical exponent of the specific heat) and with the renormalized one provided $\alpha<0$. Early renormalization group studies of systems with constrained nonfluctuating parameter were presented in Refs. $[4,8,9]$.

As it is shown in Ref.[1], the nature of a phase transition may strongly depend on the constraint imposed on the system. For the case of the unconstrained system the stability criterion for the occurrence of continuous phase transition can be found within the mean field theory. On the other hand, for the case of a constrained system a different criterion, which is distinct from the mean-field one, is calculated. This criterion results in a fluctuation-induced renormalized Heisenberg tricritical point.

The main purpose of this paper is to consider the influence of disordering upon critical behavior of a system with hidden degrees of freedom and to focus attention on a criterion for the occurrence of continuous phase transition in this system. Such disordering can be caused, e.g., by the presence of "frosen" impurities [10]. The analysis of renormalization group equations for random system with constrained nonfluctuating parameter has been presented in Ref.[11]. It was found the fixed points and shortly described the critical behavior. Here we discuss the tricritical behavior and especially a first-order transition taking into account results of [1].

\section{Renormalization group equations}

Let us consider the disordered system in which an $n$-component vector order parameter $\mathbf{S}(\mathbf{x})$ is coupled with the scalar nonfluctuating order parameter $y(\mathbf{x})$. This system can be described in momentum space by the effective Hamiltonian [11]

$$
\begin{aligned}
H= & \frac{1}{2} \int \frac{d \mathbf{q}_{1}}{(2 \pi)^{d}} \frac{d \mathbf{q}_{2}}{(2 \pi)^{d}} r\left(\mathbf{q}_{1}, \mathbf{q}_{2}\right) \mathbf{S}\left(\mathbf{q}_{1}\right) \mathbf{S}\left(\mathbf{q}_{2}\right)+ \\
& +\int \frac{d \mathbf{q}_{1}}{(2 \pi)^{d}} \frac{d \mathbf{q}_{2}}{(2 \pi)^{d}} \frac{d \mathbf{q}_{3}}{(2 \pi)^{d}} \frac{d \mathbf{q}_{4}}{(2 \pi)^{d}} u\left(\mathbf{q}_{1}, \mathbf{q}_{2}, \mathbf{q}_{3}, \mathbf{q}_{4}\right) \mathbf{S}\left(\mathbf{q}_{1}\right) \mathbf{S}\left(\mathbf{q}_{2}\right) \mathbf{S}\left(\mathbf{q}_{3}\right) \mathbf{S}\left(\mathbf{q}_{4}\right)+ \\
& +\int \frac{d \mathbf{q}_{1}}{(2 \pi)^{d}} \frac{d \mathbf{q}_{2}}{(2 \pi)^{d}} \frac{d \mathbf{q}_{3}}{(2 \pi)^{d}} \mu\left(\mathbf{q}_{1}, \mathbf{q}_{2}, \mathbf{q}_{3}\right) y\left(\mathbf{q}_{1}\right) \mathbf{S}\left(\mathbf{q}_{2}\right) \mathbf{S}\left(\mathbf{q}_{3}\right)+ \\
& +\frac{1}{2} \int \frac{d \mathbf{q}_{1}}{(2 \pi)^{d}} \frac{d \mathbf{q}_{2}}{(2 \pi)^{d}} \beta\left(\mathbf{q}_{1}, \mathbf{q}_{2}\right) y\left(\mathbf{q}_{1}\right) y\left(\mathbf{q}_{2}\right)+\int \frac{d \mathbf{q}}{(2 \pi)^{d}} h(\mathbf{q}) y(\mathbf{q})
\end{aligned}
$$

The Hamiltonian (1) can be used to study the ferromagnetic phase transition 
in the three-dimensional Hubbard model with frosen impurities. Herewith, the coupling $\mu$ is pure imaginary

$$
\mu=i\left(k T_{c}\right)^{1 / 2} U^{3 / 2} N^{\prime}\left(E_{f}\right),
$$

where $N\left(E_{f}\right)$ is the density of states on the Fermi energy $E_{f}$ and $U$ is the Coulomb potential. The study of this case is of particular interest.

Following the standard method of the renormalization group [12] and its extension to random systems [10] the recurrence relations for the potential averages over the probability distribution function can be constructed by averaging on the recurrence relations for the potentials of the Hamiltonian (1) of the inhomogenious system and for the second cumulants:

$$
\begin{aligned}
r^{\prime} & =b^{2}\{r+[4(n+2) \tilde{u}-\tilde{\Delta}+2 n z] A(r)\}, \\
\tilde{u}^{\prime} & =b^{\epsilon}\left\{\tilde{u}-K_{4} \ln b\left[4(n+8) \tilde{u}^{2}-6 \tilde{u} \tilde{\Delta}\right]\right\} \\
\tilde{\Delta}^{\prime} & =b^{\epsilon}\left\{\tilde{\Delta}-K_{4} \ln b\left[8(n+2) \tilde{u} \tilde{\Delta}-4 \tilde{\Delta}^{2}\right]\right\}, \\
z^{\prime} & =b^{\epsilon}\left\{z-K_{4} \ln b\left[8(n+2) \tilde{u} z+2 n z^{2}-2 z \tilde{\Delta}\right]\right\}, \\
w^{\prime} & =b^{\epsilon}\left\{w-K_{4} \ln b\left[8(n+2) \tilde{u} w+2 n w^{2}+4 z w-2 w \tilde{\Delta}\right]\right\},
\end{aligned}
$$

where we introduce the following symbols for second cumulants: $\Delta \sim\left\langle r r>_{c}\right.$, $\nu \sim<h>_{c}, \rho \sim<h h>_{c}$, and use the notation: $\tilde{u}=u-\frac{\mu^{2}}{2 \beta}, \tilde{\Delta}=\Delta-\frac{4 \mu \nu}{\beta}+\frac{4 \mu^{2} \rho}{\beta^{2}}$, $z=\frac{\mu^{2}}{\beta}-\frac{\mu_{0}^{2}}{\beta_{0}}, w=\frac{\mu_{0}^{2}}{\beta_{0}}$. All other definitions are standard in renormalization group theory: $K_{4}=\frac{1}{8 \pi^{2}}$ is a quality proportional to the surface area of the unit sphere in $d=4$ space, $b$ is the scale parameter and $A(r)$ is the integral of a closed loop of pair correlation functions (see, also, [13]).

In Eqs.(3-7) we separate the coefficient of the nonfluctuating parameter $y(\mathbf{q}=$ $0)$ from those of $y(\mathbf{q} \neq 0)$ because of their possible role in constraining systems $[4,8]$. We make a shift of the variable $y(\mathbf{q}=0)$ for the disappearance of the linear term in the variable $y$ on each step of the renormalization procedure. It is also necessary to write the equations for the cumulants $\nu, \nu_{0}, \rho \mu, \rho_{0} \mu_{0}$. However, the structure of the new equations is such that they do not violate the stability of fixed points, which were defined by Eqs.(3-7). Using the definition of cumulants $\Delta, \nu, \rho$ one can show that $\tilde{\Delta} \geq 0$.

Before discussing the renormalization group analysis, consider the mean-field theory results. After integrating over the nonfluctuating parameter in (1), we obtain a new effective Hamiltonian for order parameter $\mathbf{S}(\mathbf{q})$. A solution of this new integrated out effective Hamiltonian in the mean-field approximation gives the boundary conditions of instability for the Hubbard model: $\lambda_{s}-\lambda_{c}^{(0)}=0$, $\lambda_{c}^{(0)}=0$, where $\lambda_{s} \equiv u$ and $\lambda_{c}^{(0)}=\mu_{0}^{2} / 2 \beta_{0}$, for unconstrained system $\left(\lambda_{c}^{(0)}=\right.$ $\left.\lambda_{c}^{(1)}=\lambda\right)$ and $\lambda_{s}=0, \lambda_{c}^{(1)}=0$, where $\lambda_{c}^{(1)}=\mu^{2} / 2 \beta$, for the constrained system 


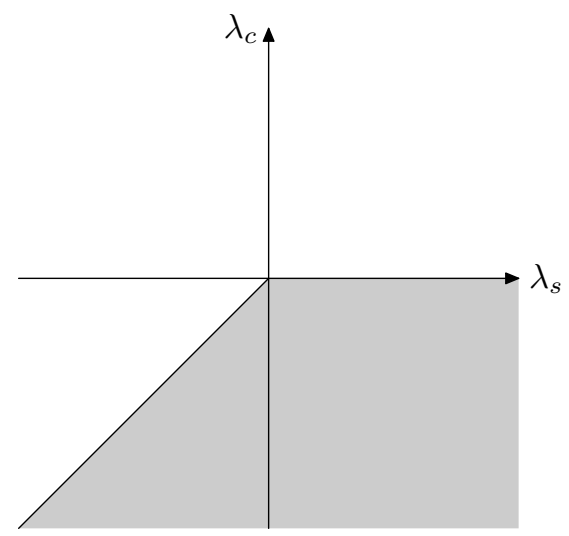

Fig. 1. Phase diagram in the mean-field approximation for the unconstrained system

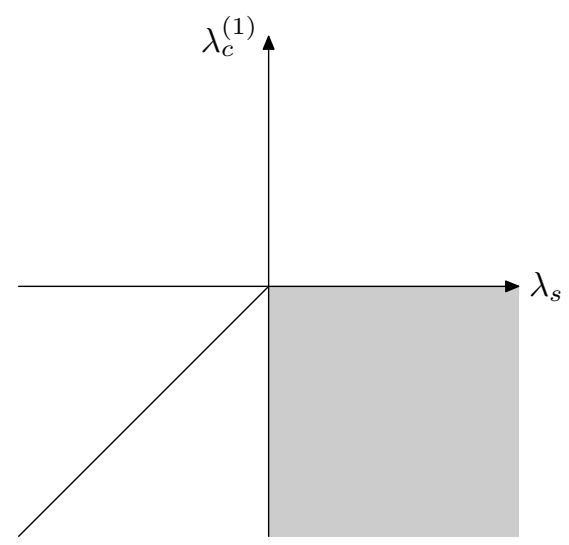

Fig. 2. Phase diagram in the mean-field approximation for the constrained system $\left(\lambda_{c}^{(0)}=0\right)$. These conditions divide the first and second order phase transition ranges in $\left(\lambda_{c}, \lambda_{c}^{(1)}\right)-\lambda_{s}$-plane (Fig. 1 and Fig. 2, see, also, [1]). Using the Stoner criterion $r_{s} \equiv 1-(U / 2) N\left(E_{f}\right)=0$, we have the condition for a continuous phase transition for the unconstrained system

$$
\frac{3\left(N^{\prime}\left(E_{f}\right)\right)^{2}}{2 N\left(E_{f}\right)}>N^{\prime \prime}\left(E_{f}\right),
$$

while the first order phase transition occurs for the contrary case. For the constrained system we have the continuous phase transition, if

$$
N^{\prime \prime}\left(E_{f}\right)<0 \text {. }
$$

According to the mean-field theory for random systems, the impurities do not influence on the stability ranges of the system.

A feature of the recurrence relations is a closed system of two equations: (4) and (5). It is easy to find the fixed points of this system. We have as usual the Gaussian fixed point $(G)$ with $\tilde{u}^{*}=\tilde{\Delta}^{*}=0$, the Heisenberg fixed point 


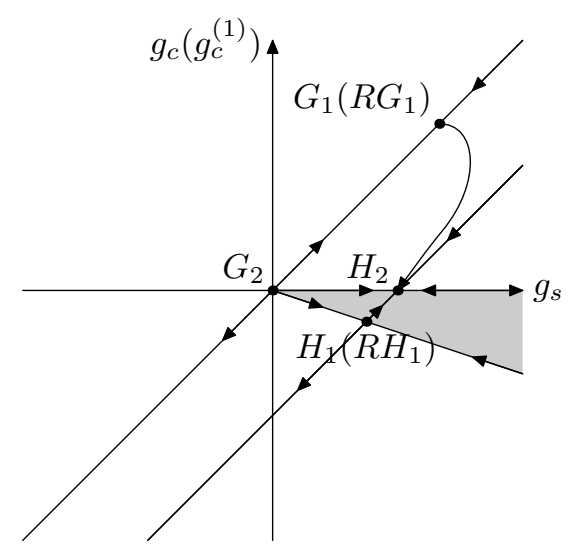

Fig. 3. Flow trajectories in the $\tilde{\Delta}=0$ plane

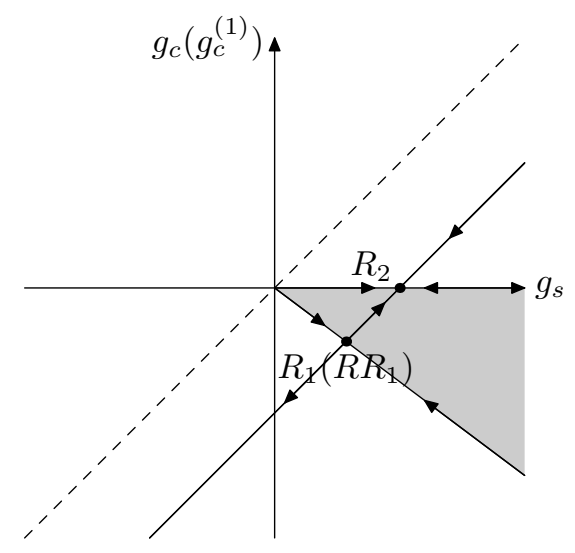

Fig. 4. Flow trajectories in the $\tilde{\Delta}=\tilde{\Delta}^{*}$ plane

$(H)$ with $\tilde{u}^{*}=\epsilon / 4 K_{4}(n+8), \tilde{\Delta}^{*}=0$, the non-physical fixed point with $\tilde{u}^{*}=0, \tilde{\Delta}^{*}=-\epsilon / 4 K_{4}$, and random fixed point $(R)$ with $\tilde{u}^{*}=\epsilon / 16 K_{4}(n-1)$, $\tilde{\Delta}^{*}=\epsilon(4-n) / 8 K_{4}(n-1)$. Using these fixed points, one can easy find the rest fixed points from the recurrence relations.

The full list of fixed points, the critical behavior, which is determined by their values, and their stability have been presented in [11]. We can see from the Table in [11] that some parameters and their selfvalues $\lambda_{i}$ change sign at $n=4$. This changing in sign strongly influences the forms of flow trajectories, and we are obliged further to discuss separately the $n<4$ and $n>4$ cases. It is convenient to use the two parameter spaces: $\left(\lambda_{s}, \lambda_{c}, \tilde{\Delta}\right)$ for the unconstrained case $\left(\lambda_{c}^{(0)}=\lambda_{c}^{(1)}=\lambda_{c}\right)$ and $\left(\lambda_{s}, \lambda_{c}^{(1)}, \tilde{\Delta}\right)$ for the constrained case $\left(\lambda_{c}^{(0)}=0\right)$. Moreover, it is turn out that the interesting fixed points are in the planes $\tilde{\Delta}=0$ and $\tilde{\Delta}=\tilde{\Delta}^{*} \neq 0$ in both spaces. Due to the relation $\tilde{u}=u-\mu^{2} / 2 \beta \equiv \lambda_{s}-\lambda_{c}^{(1)}$ all fixed points align on two parallel lines in these planes. The flow trajectories are identical in both spaces, so we can demonstrate them simultaneously in the $\tilde{\Delta}=0$ (Fig. 3) and $\tilde{\Delta}=\tilde{\Delta}^{*}$ (Fig. 4) planes in the spaces of the renormalized coupling constants $g_{s}, g_{c}^{(0)}$, and $g_{c}^{(1)}$. The notation of fixed points, which correspond to the subspace $\left(\lambda_{s}, \lambda_{c}^{(1)}, \tilde{\Delta}\right)$ are written in brackets. It is important 
to point out that fixed points with $z^{*} \equiv 2\left(\lambda_{c}^{(1)^{*}}-\lambda_{c}^{(0)^{*}}\right) \neq 0$ determine the renormalized critical behavior in accordance with [5].

One can see from Fig. 3 and Fig. 4 that the most stable fixed points and, hence, the critical behavior is independent of the existence of constraint. One point is $H_{2}$, when $n>4$, and the critical behavior is ideal for a pure system and another point is $R_{2}$, when $n<4$, and the critical behavior is determined by the presence of the frosen inhomogeneities. Thus, the behavior coincides with the one found in [10].

The critical behavior at the boundary of the stability region is determined by the fixed points $R_{1}$ (for $n<4$ ) and $H_{1}$ (for $n>4$ ) in the system without constraints, and by the "renormalized" fixed points $R R_{1}$ (for $n<4$ ) and $R H_{1}$ (for $n>4$ ) in system with constraints. It is easy to see that the boundary of the stability region in constrained systems reduces the criterion (9) for pure system to

$$
\frac{3\left(N^{\prime}\left(E_{f}\right)\right)^{2}}{2 N\left(E_{f}\right)}<\frac{\alpha_{H} / n \nu_{H}}{\epsilon /(n+8)+\alpha_{H} / n \nu_{H}} N^{\prime \prime}\left(E_{f}\right)+O(\epsilon)
$$

where $\alpha_{H}=\frac{4-n}{2(n+8)} \epsilon$ and $\nu_{H}=\frac{1}{2}+\frac{n+2}{4(n+8)} \epsilon$ are the specific heat and the correlation length critical exponents for the Heisenberg fixed point, respectively (see, also, [1]). By analogy with a pure system, one can find the corresponding criterion for a random constrained system in the form

$$
\frac{3\left(N^{\prime}\left(E_{f}\right)\right)^{2}}{2 N\left(E_{f}\right)}<\frac{\alpha_{R} / n \nu_{R}}{\epsilon / 4(n-1)+\alpha_{R} / n \nu_{R}} N^{\prime \prime}\left(E_{f}\right)+O(\epsilon),
$$

where $\alpha_{R}=\frac{n-4}{8(n-1)} \epsilon$ and $\nu_{R}=\frac{1}{2}+\frac{3 n}{32(n-1)} \epsilon$ are corresponding exponents for the random fixed point. Thus, in the constrained system the flow trajectories run away in both the pure system fixed point $H_{1}$ and the random fixed point $R_{1}$, and, hence, the critical behavior at the boundary of the stability region reduces to the tricritical behavior.

\section{The tricritical behavior}

Using the standard field theory and renormalization group method (see, for example, [14]), we obtained the next equation for the free energy in the one loop approximation

$$
\beta F(T, M)=\frac{1}{2} r_{s}(T) M^{2}+\frac{1}{4 !}\left(\lambda_{s}-\lambda_{c}^{(0)}\right) M^{4}-
$$




$$
\begin{aligned}
& -\frac{1}{2} \tilde{\Delta} M^{2} \int \frac{d \mathbf{q}_{1}}{r_{s}(T)+q^{2}}-\frac{6}{4 !} \lambda_{2} \tilde{\Delta} M^{4} \int \frac{d \mathbf{q}_{1}}{\left(r_{s}(T)+q^{2}\right)^{2}}+ \\
& +\frac{1}{2}(n-1) \int d \mathbf{q}_{1} \ln \left(1+\frac{\lambda_{1} M^{2} / 6}{r_{s}(T)+q^{2}}\right)+ \\
& +\frac{1}{2} \int d \mathbf{q}_{1} \ln \left(1+\frac{\lambda_{2} M^{2} / 6}{r_{s}(T)+q^{2}}\right),
\end{aligned}
$$

where $M=\left(\sum_{\alpha} M_{\alpha}^{2}\right)^{1 / 2}$ is the magnetization, and

$$
\lambda_{1}=\frac{1}{3}\left(\lambda_{s}-\lambda_{c}^{(0)}\right), \quad \lambda_{2}=\frac{1}{3}\left(3 \lambda_{s}-\lambda_{c}^{(0)}-2 \lambda_{c}^{(1)}\right) .
$$

Note that here we used coefficients of Eq.(1) in form: $u=\lambda_{s} / 4 !, \mu_{0}^{2} / 2 \beta_{0}=$ $\lambda_{c}^{(0)} / 4$ !, $\mu^{2} / 2 \beta=\lambda_{c}^{(1)} / 4$ !. To get the renormalized free energy, we used the theory of Refs.[14] and [1]. Finally, we have

$$
\begin{aligned}
\beta F(t, M)-\beta F(t, 0)= & \frac{1}{2} \tilde{t} M^{2}+\frac{1}{4 !}\left(\tilde{g}_{s}-\tilde{g}_{c}^{(0)}\right) M^{4}+ \\
& (n-1) f\left(t+\frac{1}{2} g_{1} M^{2}\right)+f\left(t+\frac{1}{2} g_{2} M^{2}\right),
\end{aligned}
$$

where $\tilde{t}, \tilde{g}_{s}$, and $\tilde{g}_{c}^{(0)}$ are the renormalized by frozen impurities values, $g_{1}$ and $g_{2}$ are renormalized coupling constants $\lambda_{1}$ and $\lambda_{2}$ and

$$
f(x)=\frac{1}{8} x^{2}\left(\ln x-\frac{1}{2}\right) .
$$

For the unconstrained case $g_{c}^{(0)}=g_{c}^{(1)}=g_{c}$ we have the conventional $\phi^{4}$-model with an effective coupling constant proportional to $g_{s}-g_{c}$ and the runaway flow trajectories do not intersect the boundary of the stability range. Hence, we can suggest that the phase transition remains second order. It should be noted here, that the fixed points $H_{1}$ and $R_{1}$ are not tricritical points. In particular, this fact is in accordance with the result for pure systems [1]. Also, following [1], we can look on the lines connecting the origin and fixed points $H_{1}$ and $R_{1}$ in Figs. 3 and 4 as boundary lines between two second order phase transition regions with different critical behaviors.

For the constrained case $g_{c}^{(0)} \neq g_{c}^{(1)}$ the runaway flow trajectories intersect the boundary of stability range. The critical behavior at the boundary of that stability region is determined by the points $R R_{1}(n<4)$ and $R H_{1}(n>4)$. We suppose that this means rather the smeared phase transition than the phase transition of first order, in accordance with [10] and [15]. 
In summary, we considered the influence of disordering upon the critical behavior in systems with hidden degrees of freedom taking into account the possible role of the constraint upon these degrees of freedom. It is shown that there are the tricritical fixed points in the constrained systems and we have rather smeared phase transition than the first order transition, while in the unconstrained system all phase transitions are the phase transition of the second order.

\section{References}

[1] M.L.Lyra, M.D.Coutinho-Filho, and A.M.Nemirovsky, Phys. Rev. B 48 (1993) 3755-3769.

[2] D.R.Nelson and M.E.Fisher, Phys. Rev. B 11 (1975) 1030.

[3] J.M.Kosterlitz, D.R.Nelson, and M.E.Fisher, Phys. Rev. B 13 (1976) 412.

[4] C.M.Chaves, P.Lederer, and A.A.Gomes, J.Phys. C 10 (1977) 3367.

[5] M.E.Fisher, Phys. Rev. 176 (1968) 257.

[6] Y.Imry, O.Entin-Wohlman, and D.J.Berdman, J. Phys. C 6 (1973) 2846.

[7] V.Dohm, J. Phys. C 7 (1974) L174.

[8] Y.Achiam and Y.Imry, Phys. Rev. B 12 (1975) 2768.

[9] Y.Achiam, J. Phys. C 10 (1977) 1491.

[10] T.C.Lubensky, Phys. Rev. B 11 (1975) 3573.

[11] V.M.Laptev and Yu.N.Skryabin, phys. stat. sol. (b) 91 (1979) K143.

[12] K.G.Wilson and J.Kogut, Physics Rep. C 12 (1979) 75.

[13] Yu.A.Izyumov and Yu.N.Skryabin, Statistical Mechanics of Magnetically Ordered Systems (Consultants Bureau, New York, London, 1988).

[14] D.J.Amit, Field Theory, The Renormalization Group and Critical Phenomena (World Scientific, Singapore, 1984).

[15] A.Aharony, Phys. Rev. B 12 (1975) 1038. 